\title{
Cost-Effectiveness of a Sublingual Bacterial Vaccine for the Prophylaxis of Recurrent Urinary Tract Infections
}

\author{
Pedro Carrión-López Jesus Martínez-Ruiz José Miguel Giménez-Bachs \\ Pedro Jesús Fernández-Anguita Inmaculada Díaz de Mera-Sánchez Migallón \\ Oscar Legido-Gómez Saúl Rico-Marco Marta Victoria Lorenzo-Sánchez \\ Antonio Santiago Salinas-Sánchez
}

Department of Urology, Albacete University Hospital Complex, Albacete, Spain

\author{
Keywords \\ Direct costs · Healthcare resources - Recurrent urinary tract \\ infections · Sublingual vaccine
}

\begin{abstract}
Introduction: Recurrent urinary tract infections (rUTIs) affect $5-10 \%$ of women, resulting in an enormous healthcare and society burden. Uromune ${ }^{\circledR}$ is a polybacterial sublingual vaccine with an excellent clinical benefit in rUTI prophylaxis. This study assesses the impact of sublingual vaccination on healthcare resource use and expenditures associated with this pathology. Methods: A quasi-experimental, pretestposttest, single center study including women with rUTI and vaccinated with Uromune ${ }^{\circledast}$ in real-life clinical practice was performed. Variables were the need of healthcare resources, collected prospectively during two follow-up years, and the rUTI-associated expenditure, calculated using the microcosting methodology; these were compared before and after vaccination. Results: A total of 166 women \{mean (standard deviation [SD]) urinary tract infection episodes/year 6.19 (2.15)\} were included. After vaccination, annual consultations with a primary care physician (PCP) (43.9\%), emergency room visits (71.8\%), urinary analysis (90.0\%), and ultra-
\end{abstract}

karger@karger.com www.karger.com/uin

Karger $\stackrel{\text { ' }}{5}$

GOPEN ACCESS
(C) 2022 The Author(s)

Published by S. Karger AG, Basel

This is an Open Access article licensed under the Creative Commons Attribution-NonCommercial-4.0 International License (CC BY-NC) (http://www.karger.com/Services/OpenAccessLicense), applicable to the online version of the article only. Usage and distribution for commercial purposes requires written permission. sound exams (35.6\%) decreased compared to pre-vaccination (all $p<0.001$ ). Per patient consumption in antibiotics, PCP consultations, emergency room visits, and complementary exams significantly decreased (all $p<0.02$ ), resulting in a reduction in healthcare expenditure per patient/year from mean (SD) 1,001.1 (655.0) to 497.1 (444.4) EUR. Conclusion: Sublingual bacterial vaccination with Uromune ${ }^{\circledR}$ decreased healthcare resource use and associated expenditure in women with rUTI, representing an optimal strategy to reduce rUTI-associated healthcare and economic burden.

(C) 2022 The Author(s)

Published by S. Karger AG, Basel

\section{Introduction}

Urinary tract infections (UTIs) are among the most common bacterial infections and reasons for seeking consultation. It is estimated that 150 million people worldwide, primarily women, suffer from UTIs annually. Twenty-seven percent of these women suffering an initial UTI experience, recurrent UTIs (rUTIs), with a notable impact on their quality of life. Altogether, due to the high burden to society and healthcare systems, rUTI constitutes a significant global healthcare concern [1]. 
Management of rUTI is associated with high direct and indirect costs. Direct costs encompass healthcare expenses related to medication, complementary tests, consultations to physicians, and hospital admissions, whereas indirect costs include those associated with UTI-dependent absenteeism and pathology-derived follow-up [2]. In the USA, over 11.3 million women suffer more than one UTI episode per year, resulting in a total expense of 659 USD and 936 USD million in direct and indirect costs, respectively [1].

The mainstay treatment for rUTI is antibiotic therapy, either prophylactic, postcoital, or on-demand. However, resistances to antimicrobials, generated by misuse and overuse of antibiotic therapy, are increasingly challenging their efficacy $[3,4]$. Vaccines against urinary pathogens, aimed to increase the natural immune response against uropathogens, emerge as additional nonantibiotic therapies to prevent recurrences and decrease antibiotic use [5]. Of note, their development is encouraged by current guidelines for the management of urological infections [6, 7]. Among them, mucosal bacterial vaccines, composed of either extracts or whole-cell bacteria, have shown to trigger protective immune responses and ameliorate rUTI [5, $8-10]$. In this regard, previous studies have shown that the sublingual bacterial vaccine Uromune ${ }^{\circledR}$ is effective in reducing the UTI incidence and preventing recurrence, showing a robust clinical benefit and an excellent safety profile for patients with rUTI [11-17].

Despite the proven effectiveness of sublingual bacterial vaccines in the management of rUTI, studies evaluating their impact on healthcare resource consumption and costs associated with rUTI in the Spanish real-world setting are missing. In this study, we assess the economic impact of direct healthcare costs of rUTI management and analyze changes in healthcare resource use and direct costs following sublingual vaccination with Uromune ${ }^{\circledR}$.

\section{Material and Methods}

\section{Study Design and Population}

This was a quasi-experimental, pretest-posttest, single-center study including women diagnosed with rUTI who received sublingual vaccination under real-life clinical practice and were followed-up for 1-2 years. Patients included in this study meet the established criteria for rUTI diagnosis, 2 or more UTIs in 6 months or 3 or more UTIs in 12 months [6,7]. Women requiring medical consultation at the Urology Department of Albacete University Hospital Complex (Albacete, Spain), refractory to prophylactic antibiotics and prescribed with Uromune ${ }^{\circledR}$, were consecutively included in the study. Those diagnosed with autoimmune diseases or refusing treatment with the vaccine were excluded. The study was conducted from January 2013 to December 2016.

Uromune Cost-Effectiveness in Recurrent Urinary Tract Infections
Treatment

Uromune $^{\circledR}$ is a sublingual preparation composed of a mixture of whole-cell inactivated bacteria (300 Formazin Turbidity Units; approx. $10^{9}$ bacteria/mL): Escherichia coli (V121), Klebsiella pneumoniae (V113), Enterococcus faecalis (V125), and Proteus vulgaris (V127) strains (manufacturer, Inmunotek S.L.; Spanish distributor, QPharma laboratories). In this study, either a formulation at equal amounts per bacterial strain or with individualized percentages per patient were used, according to the physicians' criteria. The vaccine was administered in two $100-\mu \mathrm{L}$ sprays on the sublingual mucosa once a day for 3 months.

\section{Objectives and Outcomes}

This study focused on the cost analysis of a previous study whose primary objective was to evaluate the effectiveness of the sublingual bacterial vaccine in the prophylaxis of rUTI [11]. Before inclusion in the study, variables were collected from medical records retrospectively (pre-vaccination). During the study, data were collected prospectively, throughout at least 1 year and up to 2 years, after starting vaccination (postvaccination period). Clinical variables to assess effectiveness were described elsewhere [11]. Healthcare resource use-related variables included antibiotic treatments, urine cultures [11], primary care physician (PCP) visits, urologist visits, emergency room admissions, hospitalizations (including duration), and other complementary tests (i.e., urine laboratory analysis and ultrasound exams).

rUTI-dependent expenditure was calculated using the microcosting methodology based on (i) assessing the healthcare resources used in rUTI patient care, (ii) estimating the need for each component, and (iii) assigning a unit cost (EUR) per resource. Costs were based on fees established according to the April 06, 2010, resolution, obtained from the Direction of the Regional division (Albacete) of the Department of Health (online suppl. Table S1; for all online suppl. material, see www.karger.com/doi/10.1159/000521772). Vaccine expenses were also included.

\section{Statistical Analysis}

A sample size of 149 women was deemed necessary to estimate a decrease in UTI incidence of 3 episodes/year with a risk of $\alpha<0.05$ and a $95 \%$ confidence, considering a potential dropout of $10 \%$. Categorical variables were described as frequencies and percentages, while quantitative variables as mean and standard deviation (SD) or $95 \%$ confidence interval (CI). Changes following vaccination were calculated as mean (95\% CI) of individual percentages of increase and decrease per year compared to before treatment. Paired Student's $t$ test or the Wilcoxon signed-rank test was used for intragroup analysis (pre- vs. postvaccination). A level of significance of $p<0.05$ was established for all tests performed (two-sided). Statistical analyses were performed using SPSS software (version 20).

\section{Results}

Impact of Recurrent UTIs on Healthcare Resource Use

A total of 166 women suffering from rUTI (mean age of 62.3 years [SD 18.4]) were included in the study. Patients had been followed up at the participating center for a mean of 2.70 years (SD 1.60) prior to inclusion, pre- 
Table 1. Use of healthcare resources per patient/year

\begin{tabular}{|c|c|c|c|c|c|}
\hline & \multicolumn{2}{|c|}{ Before vaccination } & \multicolumn{2}{|c|}{ After vaccination } & \multirow[t]{2}{*}{$p$ value $^{1}$} \\
\hline & mean (SD) & $95 \% \mathrm{Cl}$ & mean (SD) & $95 \% \mathrm{Cl}$ & \\
\hline \multicolumn{6}{|l|}{ Antibiotic treatments ${ }^{2}, n$} \\
\hline Prescriptions & $7.34(4.05)$ & $6.72-7.96$ & $2.89(3.9)$ & $2.28-3.49$ & $<0.001$ \\
\hline Distinct antibiotics used & $2.12(1.11)$ & $1.95-2.29$ & $1.22(1.09)$ & $1.05-1.39$ & $<0.001$ \\
\hline \multicolumn{6}{|c|}{ Consultations and specialized care } \\
\hline PCP & $4.69(3.22)$ & $4.20-5.19$ & $2.05(2.66)$ & $1.65-2.46$ & $<0.001$ \\
\hline Urologist & $0.66(0.38)$ & $0.54-0.66$ & $1.47(0.81)$ & $1.34-1.59$ & $<0.001$ \\
\hline Emergency room visits & $0.41(0.71)$ & $0.30-0.52$ & $0.13(0.37)$ & $0.08-0.19$ & $<0.001$ \\
\hline Hospitalizations & $0.05(0.20)$ & $0.02-0.08$ & NA & NA & $N A^{3}$ \\
\hline \multicolumn{6}{|l|}{ Complementary exams } \\
\hline Ultrasound & $0.37(0.50)$ & $0.30-0.44$ & $0.12(0.29)$ & $0.08-0.17$ & $<0.001$ \\
\hline Urine laboratory analysis & $1.47(1.40)$ & $1.25-1.69$ & $0.89(1.37)$ & $0.68-1.10$ & $<0.001$ \\
\hline
\end{tabular}

sented an annual mean of 6.19 (SD 2.15) UTI episodes, and required a total of 1,566 urine cultures. Patients were prescribed a total of 3,120 antibiotic treatments, and most of them $(n=119,71.7 \%)$ were treated with $\geq 4$ different antibiotics [11].

During the pre-vaccination period, all women consulted their PCPs at least once, with 12 (7.2\%) women attending over 10 times annually. Urologist visits were required by 153 (92.2\%) patients. Sixty-six (39.7\%) and 17 (10.2\%) women were admitted to emergency rooms and hospital wards, respectively. At least once before treatment, 99 (59.6\%) patients needed ultrasound exams, and $148(89.2 \%)$ required urine laboratory analyses, with 9 (5.4\%) women needing $\geq 10$ tests. Healthcare resource use per patient/year in the pre-vaccination period is summarized in Table 1.

\section{Impact of Bacterial Vaccination on Healthcare}

\section{Resource Use Associated with rUTI}

All 166 patients included in the study completed the 3 -month course and were followed up for a mean of 1.71 years (SD 0.28) (evaluable population). After vaccination, the number of UTI episodes and urine cultures per year significantly decreased by $54.6 \%$ and $37.5 \%$, respectively, compared to the pre-vaccination. The annual use of antibiotics, measured as the number of packages and the number of different antibiotics used $(60.7 \%$ and $42.5 \%$ drop, respectively), was overall reduced (Table 1) [11].

Consistent with the decrease of UTI episodes, the annual need for any healthcare resources significantly de- creased following vaccination, except for the number of hospital admissions, which were barely required (Table 1). The mean percentage decrease in PCP visits per patient/year was $43.9 \%$ (95\% CI 32.3-55.6), although these consultations remained unchanged in 34 (20.5\%) women. The highest reduction was for emergency rooms admissions, achieving a 71.8\% (95\% CI, 58.884.8) decline compared to pre-vaccination. Regarding urologist visits, 151 (91.0\%) women required increased number of consultations, resulting in a mean percentage increase of $214.5 \%$ (95\% CI, 171.0-258.1) per patient/year. After vaccination, only $4(2.4 \%)$ women required hospitalizations, with significantly shorter mean duration: 0.23 days (SD 0.90) and 0.03 (SD 0.20) $(p=$ 0.001 ) before and after vaccination, respectively. Complementary tests, including urine laboratory analyses and ultrasound exams, significantly decreased by mean 90.0\% (95\% CI 65.3-114.7) and 35.6\% (95\% CI 10.451.7), respectively.

\section{Impact of Bacterial Vaccination on Direct Healthcare} Costs Associated with rUTI

Before vaccination, the mean direct annual cost per patient was 1,001.1 EUR (SD 655.2), distributed as follows: PCP consultations (64.1\%), specialized care (24.6\%), complementary exams (6.6\%), and antibiotic treatments (4.7\%) (Tables 2, 3). Considering the number of UTI episodes and their associated costs (online suppl. Table S1), the expenditure per UTI episode before vaccination was estimated at $161.8 \mathrm{EUR}$. 
Table 2. Annual costs of consultations to healthcare system due to urinary infectious episodes

\begin{tabular}{|c|c|c|c|c|c|}
\hline & \multicolumn{2}{|c|}{ Before vaccination } & \multicolumn{2}{|c|}{ After vaccination } & \multirow[t]{2}{*}{$p$ value } \\
\hline & mean (SD) & $95 \% \mathrm{Cl}$ & mean (SD) & $95 \% \mathrm{Cl}$ & \\
\hline PCPs (EUR/patient) & $640.9(439.0)$ & $573.5-708.3$ & $280.9(364.1)$ & $225.1-336.7$ & 0.022 \\
\hline Specialized care (EUR/patient) & $246.2(402.3)$ & $184.3-308.0$ & $160.0(139.7)$ & $138.5-181.5$ & $<0.001$ \\
\hline Urologist & $99.6(81.2)$ & $87.7-112.2$ & $129.0(71.6)$ & 117.9-140.0 & ns \\
\hline Emergency room visits & $51.8(88.7)$ & $38.2-65.5$ & $17.3(46.1)$ & $10.2-24.4$ & $<0.001$ \\
\hline Hospitalizations & $94.6(363.0)$ & $38.8-150.5$ & $13.7(96.1)$ & $1.15-28.5$ & $<0.001$ \\
\hline
\end{tabular}

ns, not significant. ${ }^{1}$ Paired Student's $t$ test or the Wilcoxon signed-rank test comparing results before and after vaccination, following normal distribution assessment.

Table 3. Annual costs of complementary tests and exams due to UTIS

\begin{tabular}{|c|c|c|c|c|c|}
\hline & \multicolumn{2}{|c|}{ Before vaccination } & \multicolumn{2}{|c|}{ After vaccination } & \multirow[t]{2}{*}{$p$ value $^{1}$} \\
\hline & mean (SD) & $95 \% \mathrm{Cl}$ & mean (SD) & $95 \% \mathrm{Cl}$ & \\
\hline Urine laboratory analysis & $2.18(2.02)$ & $1.78-2.42$ & $1.28(1.90)$ & $0.98-1.58$ & $<0.001$ \\
\hline Urine cultures & $53.9(51.1)$ & $46.1-61.7$ & $33.8(28.8)$ & $28.8-38.8$ & $<0.001$ \\
\hline Ultrasounds & $10.1(12.5)$ & $8.2-12.0$ & $3.40(8.00)$ & $2.19-4.68$ & $<0.001$ \\
\hline Total & $66.1(57.1)$ & $57.4-74.9$ & $38.5(33.7)$ & $33.3-43.6$ & $<0.001$ \\
\hline
\end{tabular}

${ }^{1}$ Paired Student's $t$ test or the Wilcoxon signed-rank test comparing results before and after vaccination, following normal distribution assessment.

Following sublingual vaccination, the mean annual cost on antibiotic treatment/patient significantly decreased from 42.7 (SD 24.0) to 16.5 EUR (SD 20.0) ( $p<$ 0.001 ), albeit with different distribution depending on the antibiotic type (Fig. 1). The use of fosfomycin involved the highest burden, followed by norfloxacin and amoxicillin/clavulanic acid. Of note, the mean number of antibiotic-resistant $E$. coli infections was higher in women who failed to reduce antibiotic expenditure (3.9, SD 2.7) compared to those who successfully did $(2.7, \mathrm{SD} 2.5)(p=$ $0.031)$.

Annual expenses associated with PCP visits significantly decreased by mean 359.9 EUR (SD 518.9; \% decrease: $56.0 \%, 95 \%$ CI 44.4-76.3). Likewise, total expenditure associated with specialized care per patient/year significantly declined by $51.2 \%$ (SD 177.8 ; 95\% CI $23.4-$ 79.1) compared to pre-vaccination (86.1 EUR [SD 372.1]) (Table 2). Comparison of individual resources before and after treatment showed that reduced expenses in emergency room visits and hospitalizations $(p<0.001$ for all comparisons) accounted for this overall reduction, whereas a modest increase in costs of urologist consultations lacked statistical significance (Table 2).

Uromune Cost-Effectiveness in Recurrent Urinary Tract Infections
Individual costs of urine laboratory analysis, urine cultures, and ultrasounds significantly decreased by mean 0.82 (SD 2.75), 20.1 (SD 23.2), and 6.7 EUR (SD 18.0) per patient/year ( $p<0.001$ for all tests), respectively (Table 3 ). Overall, this represents a $61.2 \%(95 \%$ CI $56.7-68.1)$ decrease in complementary tests $(p<0.001)$.

In summary, the direct annual cost per patient suffering from rUTI prior to inclusion in the study were 1,001.1 EUR (SD 655.0) compared to 497.1 EUR (SD, 444.4) after vaccination. Considering the specific expenses of sublingual vaccination (172.39 EUR/treatment), the global UTI-associated healthcare expenditure per patient/year following vaccination was 669.1 EUR ( $p=0.001$, compared to the pre-vaccination period). Altogether, the decrease in healthcare resource use was associated with an overall reduction in the costs driven by rUTI.

\section{Discussion}

Results from this observational, prospective study including women with rUTI demonstrated a significant clinical, healthcare, and economic impact of this pathol- 


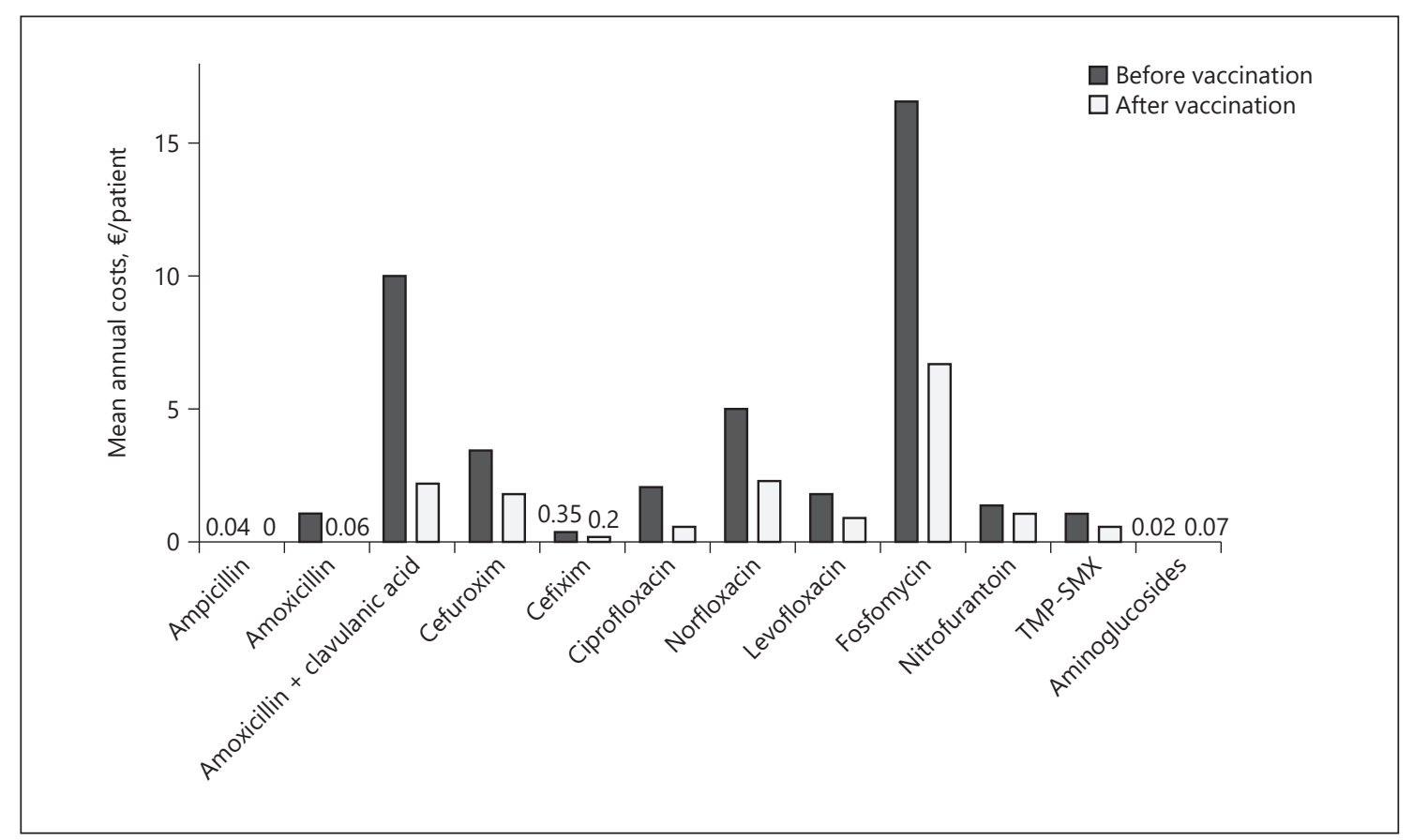

Fig. 1. Mean costs per patient/year (EUR) of the indicated antibiotic treatments before and after vaccination.

ogy due to their associated direct costs, primarily from medical consultations. The decrease in frequency of urinary infections following vaccination with the sublingual bacterial vaccine (Uromune ${ }^{\circledR}$ ) resulted in a significantly reduced need of healthcare resources. Likewise, a marked drop in healthcare-associated expenses was found, including those driven by antibiotic use, PCP consultations, specialized care, and complementary tests, leading to a significant global reduction in expenditures associated with rUTI.

Similar to this study, previous studies assessing the economic burden of UTIs in different countries have shown a substantial impact of these infections on society and healthcare systems. However, these studies have used heterogeneous methods and epidemiological criteria to assess distinct costs (i.e., productivity losses and intangible costs) at different levels (i.e., national, regional, and hospital) and in heterogeneous populations, thus precluding direct comparisons between studies [18-21]. Similar to this study, a previous large study $(2,700 \mathrm{pa}-$ tients) in patients with rUTI from several European countries found that the most considerable impact on economic costs was due to consultations followed by antibiotic therapies [21]. In Germany, the number of annual antibiotic prescriptions per patient is 3.36, representing an estimated annual cost of 54.7 EUR for the healthcare system [21], similar to the $42.7 \mathrm{EUR} /$ patient/ year calculated in this study. The micro-costing method used here assesses the economic costs of healthcare interventions accurately. This method has been an advantage to assess costs of new interventions, especially with highly variable outcomes between patients, and to estimate the real costs for the healthcare system and society [22].

Antimicrobials may be given as continuous low-dose prophylaxis for longer periods (3-6 months) or as postcoital prophylaxis as both regimens reduce the rate of rUTI. Regimens include nitrofurantoin $50 \mathrm{mg}$ or $100 \mathrm{mg}$ once daily, fosfomycin trometamol 3 g every 10 days, trimethoprim (TMP) $100 \mathrm{mg}$ once daily or $250 \mathrm{mg}$, or cefaclor $250 \mathrm{mg}$ once daily [23]. However, treatment decisions regarding UTIs should be based on the knowledge of the susceptibility profile of the uropathogens causing infections in the community against antimicrobials. TMP $100 \mathrm{mg}$ should only be considered as firstline drug in areas with known resistance rates to E. coli of $<20 \%$ [23].

Previous studies have also evaluated the cost-effectiveness of different therapeutic strategies for rUTI. Regarding antibiotic treatment, a cost minimization study conducted in Canada concluded that fosfomycin was safe and efficacious to treat UTI, owing to a low resistance profile, 
single-dose administration, and similar costs of medication [24]. A cost analysis study assessing TMP to treat rUTI showed that this antibiotic was the most effective option if antibiotic resistance remained below $30 \%$. However, a single administration of fosfomycin showed increased cost-effectiveness if resistance extended 30\%, underscoring the importance of characterizing local resistances to optimize treatment cost-effectiveness [25]. Studies comparing antimicrobial versus nonantibiotic therapies reported mean annual costs of 50 USD for Dmannose compared to 40 USD, 60 USD, and 190 USD for self-started, postcoital, and continuous antibiotic use, respectively [26]. Conversely, cranberry prophylaxis failed to be cost-effective compared to TMP sulfamethoxazole prophylaxis, regardless of the impact of antimicrobial resistance [27]. Even though healthcare expenditures of the sublingual bacterial vaccination group were not compared in parallel to antibiotic-receiving individuals in this study, the cost-effectiveness between both strategies may be extrapolated from the comparison of the post- versus pre-vaccination period as antibiotic prophylaxis was the gold-standard strategy for these patients before inclusion in the study.

Previous studies have demonstrated the clinical benefit of sublingual vaccination with Uromune ${ }^{\circledR}$ in different countries [11, 13-17]. Furthermore, a recent comprehensive systematic review of these studies concluded that the sublingual vaccine significantly benefits women who experience rUTI by preventing recurrences, with an excellent safety profile [12]. However, analysis of its cost-effectiveness remained unassessed to date. In this study, the decreased need and costs of direct healthcare resulting from the treatment with the sublingual vaccine objectively and additionally support the use of this nonantibiotic therapeutic option to minimize the high economic burden of rUTI.

The results from this study should be interpreted in the context of limitations of different nature. Similar to other pharmacoeconomic studies, this study lacked economic evaluations from a social perspective, including minor costs incurred by patients and work absenteeism. Furthermore, its pretest-posttest quasi-experimental design lacked a control group, precluding correlations between results and the treatment under evaluation but just a longitudinal analysis. Regarding the extrapolation of the results, rates of the different healthcare resources, particularly those of antibiotic therapy, are heterogeneous among different countries, precluding direct international comparisons [28]. Even though the evaluation of rUTIs economic impact and comparison between different thera- peutic options with increased accuracy would require further studies, results from this study comparing pre- and posttreatment data are valuable to provide useful information regarding cost-effectiveness of sublingual vaccination with Uromune ${ }^{\circledR}$.

\section{Conclusion}

The results from this study underscore the high economic impact of rUTI and show that treatment with the sublingual bacterial vaccine Uromune ${ }^{\circledR}$ decreased healthcare resource use and associated costs in women with rUTI. Considering the clinical impact of this pathology due to their high prevalence, high cumulative costs, and antimicrobial resistance, the use of the sublingual vaccination emerges as an optimal strategy to reduce rUTIdependent healthcare and economic burden.

\section{Statement of Ethics}

All participating patients provided written informed consents before their inclusion in the study, which was conducted in accordance with the Local Personal Data Protection Law (LOPD 15/1999). The study protocol was exempt from approval by the Ethics Committee of the participating center because the sublingual bacterial vaccine used was a drug approved by local authorities (Spanish Agency of Medicines and Medical Devices) for the prevention of rUTI. In addition, as it was a study based on clinical practice, no approval was requested from the center's Ethics Committee, and only written informed consent from the patients were included. The first patient was included in January 2013, months before the Declaration of Helsinki (Fortaleza, Brazil, October 2013), in which all research studies that included human subjects had to register in a public access database before recruiting the first subject.

\section{Conflict of Interest Statement}

The authors have no relevant affiliations or financial involvement with any organization or entity with a financial interest or financial conflict with the subject matter or materials discussed in the manuscript.

\section{Funding Sources}

No funding was received to carry out this study. 


\section{Author Contributions}

P.C.-L. conceived and designed the clinical trial. P.C.-L., J.M.R., J.M.G.-B., P.J.F.-A., I.D.M.-S.M., O.L.-G., S.R.-M., and M.V.L.S. collected clinical data as study investigators. P.C.-L. and A.S.S.S. performed statistical analysis, interpreted data, and wrote the manuscript. All the authors reviewed and revised the manuscript prior to submission.

\section{Data Availability Statement}

The data that support the findings of this study are available from https://sanidad.castillalamancha.es/sites/sescam.castillalamancha.es/files/documentos/legislacion/20131020/precios.pdf.

\section{References}

1 Foxman B. Epidemiology of urinary tract infections: incidence, morbidity, and economic costs. Dis Mon. 2003 Feb;49(2):53-70.

2 Scott R. The direct medical costs of healthcare-associated infections in US hospitals and the benefits of prevention. 2009.

3 Tacconelli E, Magrini N. Global priority list of antibiotic resistant bacteria to guide research, discovery, and development of new antibiotics. 2017.

4 Naber KG, Schito G, Botto H, Palou J, Mazzei T. Surveillance study in Europe and Brazil on clinical aspects and antimicrobial resistance epidemiology in females with cystitis (ARESC): implications for empiric therapy Eur Urol. 2008 Nov 1;54(5):1164-75.

5 Sihra N, Goodman A, Zakri R, Sahai A, Malde S. Nonantibiotic prevention and management of recurrent urinary tract infection. Nat Rev Urol. 2018;15(12):750-76.

6 Anger J, Lee U, Ackerman A, Chou R, Chughtai B, Clemens J, et al. Recurrent uncomplicated urinary tract infections in women: AUA/CUA/SUFU guideline. J Urol. 2019; 202(2):282-9.

7 Bonkat G, Bartoletti R, Bruyère F, Cai T, Geerlings SE, Köves B, et al. EAU guidelines for the management of urinary and male genital tract infections. Eur Urol. 2020 Nov:1-65.

8 O'Brien VP, Hannan TJ, Nielsen HV, Hultgren SJ. Drug and vaccine development for the treatment and prevention of urinary tract infections. Microbiol Spectr. 2016 Feb;4(1).

9 Prattley S, Geraghty R, Moore M, Somani BK. Role of vaccines for recurrent urinary tract infections: a systematic review. Eur Urol Focus. 2020;6(3):593-604.

10 Aziminia N, Hadjipavlou M, Philippou Y, Pandian SS, Malde S, Hammadeh MY. Vaccines for the prevention of recurrent urinary tract infections: a systematic review. BJU Int. 2019;123(5):753-68.

11 Carrión-López P, Martínez-Ruiz J, LibránGarcía L, Giménez-Bachs JM, Pastor-Navarro H, Salinas-Sánchez AS. Analysis of the efficacy of a sublingual bacterial vaccine in the prophylaxis of recurrent urinary tract infection. Urol Int. 2020;104(3-4):293-300.

12 Nickel JC, Saz-Leal P, Doiron RC. Could sublingual vaccination be a viable option for the prevention of recurrent urinary tract infection in Canada? A systematic review of the current literature and plans for the future. Can Urol Assoc J. 2020 May 29;14(8): 281-7.

13 Ramírez Sevilla C, Gómez Lanza E, Manzanera JL, Martín JAR, Sanz MÁB. Active immunoprophyilaxis with uromune ${ }^{\circ}$ decreases the recurrence of urinary tract infections at three and six months after treatment without relevant secondary effects. BMC Infect Dis. 2019 Oct 28;19(1):901.

14 Yang B, Foley S. First experience in the UK of treating women with recurrent urinary tract infections with the bacterial vaccine Uromune ${ }^{\oplus}$. BJU Int. 2018 Feb 1;121(2):289-92.

15 Lorenzo-Gómez MF, Padilla-Fernández B, García-Criado FJ, Mirón-Canelo JA, Gil-Vicente A, Nieto-Huertos A, et al. Evaluation of a therapeutic vaccine for the prevention of recurrent urinary tract infections versus prophylactic treatment with antibiotics. Int Urogynecol J. 2013 Jan;24(1):127-34.

16 Lorenzo-Gómez MF, Padilla-Fernández B, García-Cenador MB, Virseda-Rodríguez ÁJ, Martín-García I, Sánchez-Escudero A, et al. Comparison of sublingual therapeutic vaccine with antibiotics for the prophylaxis of recurrent urinary tract infections. Front Cell Infect Microbiol. 2015;5:50.

17 Sánchez-Ramón S, Fernández-Paredes L, SazLeal P, Diez-Rivero CM, Ochoa-Grullón J, Morado C, et al. Sublingual bacterial vaccination reduces recurrent infections in patients with autoimmune diseases under immunosuppressant treatment. Front Immunol. 2021; 12:675735.

18 François M, Hanslik T, Dervaux B, Le Strat Y, Souty C, Vaux S, et al. The economic burden of urinary tract infections in women visiting general practices in France: a cross-sectional survey. BMC Health Serv Res. 2016;16(a):365.
19 Callan A, O'Shea E, Galvin S, Duane S, Corry $\mathrm{O}$, SIMPle Team T, et al. The economic cost of urinary tract infections in the community: results from Ireland. Value Health. 2014 Nov; 17(7):A468.

20 Foxman B. Urinary tract infection syndromes: occurrence, recurrence, bacteriology, risk factors, and disease burden. Infect Dis Clin North Am. 2014 Mar 1;28(1):1-13.

21 Wagenlehner F, Wullt B, Ballarini S, Zingg D, Naber KG. Social and economic burden of recurrent urinary tract infections and quality of life: a patient web-based study (GESPRIT). Expert Rev Pharmacoecon Outcomes Res. 2018 Jan 2;18(1):107-17.

$22 \mathrm{Xu}$ X, Grossetta Nardini HK, Ruger JP. Micro-costing studies in the health and medical literature: protocol for a systematic review. Syst Rev. 2014 May 21;3:47.

23 Bonkat G, Bartoletti R, Bruyére F, Cai T, Geerling SE, Köves B, et al. Guidelines on urological infections. Arnhem: European Asociation of Urology; 2021.

24 Perrault L, Dahan S, Iliza AC, LeLorier J, Zhanel GG. Cost-effectiveness analysis of fosfomycin for treatment of uncomplicated urinary tract infections in Ontario. Can J Infect Dis Med Microbiol. 2017;2017:6362804.

25 Sadler S, Holmes M, Ren S, Holden S, Jha S, Thokala P. Cost-effectiveness of antibiotic treatment of uncomplicated urinary tract infection in women: a comparison of four antibiotics. BJGP Open. 2017 Oct 4;1(3):bjgpopen17X101097.

26 Gaitonde S, Malik RD, Zimmern PE. Financial burden of recurrent urinary tract infections in women: a time-driven activity-based cost analysis. Urology. 2019 Jun 1;128:47-54.

27 Bosmans JE, Beerepoot MAJ, Prins JM, ter Riet G, Geerlings SE. Cost-effectiveness of cranberries vs antibiotics to prevent urinary tract infections in premenopausal women: a randomized clinical trial. PLoS One. 2014 Apr 4;9(4):e91939.

28 Wagner JL, McCarthy E. International differences in drug prices. Annu Rev Public Health. 2004 Apr 11;25(1):475-95. 